\title{
molecules
}

ISSN 1420-3049

www.mdpi.com/journal/molecules

Article

\section{A Phytoanticipin Derivative, Sodium Houttuyfonate, Induces in Vitro Synergistic Effects with Levofloxacin against Biofilm Formation by Pseudomonas aeruginosa}

\author{
Jing Shao, Huijuan Cheng *, Changzhong Wang and Yan Wang \\ Laboratory of Microbiology and Immunology, School of Chinese and Western Integrative Medicine, \\ Anhui University of Traditional Chinese Medicine, Hefei 230038, China; \\ E-Mails: ustcsj@126.com (J.S.); ahwcz63@sina.com (C.W.); abudao2002@163.com (Y.W.) \\ * Author to whom correspondence should be addressed; E-Mail: chenghuijuan53@126.com; \\ Tel.: +86-551-516-9204; Fax: +86-551-516-9192.
}

Received: 9 August 2012; in revised form: 17 September 2012 / Accepted: 18 September 2012 / Published: 20 September 2012

\begin{abstract}
Antibiotic resistance has become the main deadly factor in infections, as bacteria can protect themselves by hiding in a self-constructed biofilm. Consequently, more attention is being paid to the search for "non-antibiotic drugs" to solve this problem. Phytoanticipins, the natural antibiotics from plants, could be a suitable alternative, but few works on this aspect have been reported. In this study, a preliminary study on the synergy between sodium houttuyfonate (SH) and levofloxacin (LFX) against the biofilm formation of Pseudomonas aeruginosa was performed. The minimal inhibitory concentrations (MIC) of LFX and SH, anti-biofilm formation and synergistic effect on Pseudomonas aeruginosa, and quantification of alginate were determined by the microdilution method, crystal violet (CV) assay, checkerboard method, and hydroxybiphenyl colorimetry. The biofilm morphology of Pseudomonas aeruginosa was observed by fluorescence microscope and scanning electric microscope (SEM). The results showed that: (i) LFX and SH had an obvious synergistic effect against Pseudomonas aeruginosa with MIC values of $0.25 \mu \mathrm{g} / \mathrm{mL}$ and $128 \mu \mathrm{g} / \mathrm{mL}$, respectively; (ii) $1 / 2 \times$ MIC SH combined with $2 \times$ MIC LFX could suppress the biofilm formation of Pseudomonas aeruginosa effectively, with up to $73 \%$ inhibition; (iii) the concentration of alginate decreased dramatically by a maximum of $92 \%$ after treatment with the combination of antibiotics; and (iv) more dead cells by fluorescence microscope and more removal of extracellular polymeric structure (EPS) by SEM were observed after the combined treatment of LFX and SH. Our experiments demonstrate the promising future of this potent antimicrobial agent against biofilm-associated infections.
\end{abstract}


Keywords: biofilm; sodium houttuyfonate; levofloxacin; phytoanticipin; Pseudomonas aeruginosa

\section{Introduction}

The biofilm theory was first proposed by Costerton [1] in 1978, and since then, people have realized that when bacteria are in the biofilm mode it makes them less sensitive to antibacterial agents, leading to high mortality due to repetitious infections [2]. Biofilmed bacteria are enveloped by polysaccharides, proteins, nuclear acids, etc. secreted by bacteria to protect them from antimicrobial agents; the biofilm is a self-defensive mechanism, and a structural and dynamical complex system [2]. Pseudomonas aeruginosa is one of the most widely-studied opportunistic human pathogens, and its biofilm is the main cause of many clinical infections, including chronic skin wounds [3], and the characteristic chronic lung infections of cystic fibrosis (CF) [4]. The biofilm formation tends to hinder enclosed bacterium from being killed, which is possibly related to different properties such as antimicrobial diffusion, physiological activity of inner and outer bacteria, quorum sensing, etc. [5].

To suppress the biofilm formation, many natural [6,7] and synthesized substances [8,9] have been explored. However, single antibiotic treatment is apt to induce more or less antibiotic tolerance, and cannot fully eradicate Pseudomonas aeruginosa biofilms. In terms of this problem, the combination of two antibiotics is emerging as an effective way to enhance the efficiency of biofilm inhibition and removal, and many antibiotics of microbial derivatives and metabolites have been applied in combination treatments $[7,10]$.

Different from the traditional microbial antibiotics, the phytoanticipins [11], preexisting antimicrobial agents of medicinal plants that act against pathogen and insect invasions [12], present enormous potential for biofilm eradication and anti-pathogenic activity as a strong complement to microbial antibiotics [13]. As compared with antibiotics from microbes, the phytoanticipins have the advantages of lower toxicity, more broad-spectrum antibacterial activities, and more importantly, much lower bacterial resistance for a long-time dosage.

Houttuynin [i.e., decanoyl acetaldehyde, $\mathrm{CH}_{3}\left(\mathrm{CH}_{2}\right)_{8} \mathrm{COCH}_{2} \mathrm{CHO}$ ], first isolated by Kosuge in 1952 [14], is one of the main and effective phytoanticipins extracted from Houttuynia cordata Thunb (Saururaceae family) with antimicrobial [15], antiviral [16], and anti-inflammatory activities [17]. Sodium houttuyfonate [ $\mathrm{SH}, \mathrm{CH}_{3}\left(\mathrm{CH}_{2}\right)_{8} \mathrm{COCH}_{2} \mathrm{CHOHSO}_{3} \mathrm{Na}$ ] is a derivative of houttuynin made by compounding with sodium bisulfite, soluble in hot water and alkaline solutions, but insoluble in chloroform and benzene, which exhibits broad-spectrum antibacterial activities, inhibits myocardial hypertrophy, prevents cardiac fibrosis, etc. $[15,18]$. It has been reported that $\mathrm{SH}$ and its homologues were more effective in suppressing Gram-positive $\left(\mathrm{G}^{+}\right)$bacteria, e.g., Staphylococcus aureus and Bacillus subtilis, than Gram-negative $\left(\mathrm{G}^{-}\right)$bacteria, e.g., Escherichia coli and Pseudomonas aeruginosa [19]. However, the limited reports were largely concerned with the inhibition of planktonic bacteria, and there have been no works reporting the control and removal of bacterial biofilms and the relevant encapsulated bacteria. 
In this work, we firstly used $\mathrm{SH}$ as a sole antimicrobial agent to investigate its suppression of Pseudomonas aeruginosa biofilm and alginate, one of the main component of the biofilm. Then, we employed SH as a synergistic agent for levofloxacin (LFX) to evaluate its anti-pathogenic activity on the formation of Pseudomonas aeruginosa biofilm.

\section{Results and Discussion}

\subsection{MICs of LFX and SH on Pseudomonas aeruginosa}

The minimal inhibitory concentrations (MICs) of SH and LFX were determined by the microdilution method, and they were $512 \mu \mathrm{g} / \mathrm{mL}$ and $2 \mu \mathrm{g} / \mathrm{mL}$, respectively. According to the definition of fractional inhibitory concentration index (FICI) [20], the FICI of SH and LFX could be calculated by:

$$
\mathrm{FICI}=\frac{\text { MIC of SH in combination }}{\text { MIC of SH alone }}+\frac{\text { MIC of LFX in combination }}{\text { MIC of LFX alone }}
$$

in which MICs of LFX and SH in combination were $0.25 \mu \mathrm{g} / \mathrm{mL}$ and $128 \mu \mathrm{g} / \mathrm{mL}$, respectively. Thus, FICI was 0.375 here, i.e., less than 0.5 , thus exhibiting a relative strong synergy of SH and LFX.

In our experiments, we begin to administrate in the medium after $24 \mathrm{~h}$ incubation. This medication mode mainly takes clinical applications into account. As biofilm forms, the infected focus will induce inflammation, and relevant medications commence. In other words, the biofilm may just be in the state of attachment (see Section 2.2) when the antimicrobial agents are applied.

\subsection{Biofilm Suppression by SH Alone and in Combination with LFX}

By numerous repeatable proteomic analyses, Sauer et al. put forward five stages of biofilm formation, namely: (i) reversible attachment ( $>0 \mathrm{~min})$; (ii) irreversible attachment (2 h); (iii) maturation-1 (3 days); (iv) maturation-2 (6 days); and (v) dispersion (9-12 days) [21]. In terms of this rule, we chose $1 \mathrm{~d}, 3 \mathrm{~d}$ and $7 \mathrm{~d}$ as three time-points to observe the biofilm suppression and removal after medication.

Figure 1 shows control of SH alone on biofilm formation of Pseudomonas aeruginosa. In the 1st day (Figure 1A), $1 / 2 \times$ MIC SH has nearly no effect on biofilm growth as compared with that in the control group, while $1 \times$ MIC and $2 \times$ MIC SH have obvious suppressive effects on biofilm $(p<0.01$, $\mathrm{n}=4$ ). On the 3rd day (Figure 1B), there is no statistical significance of the impact by $1 / 2 \times \mathrm{MIC} \mathrm{SH}$ treatment on biofilm, whereas, the growth of biofilm with $2 \times$ MIC SH treatment $(p=0.000, \mathrm{n}=4)$ decreases even more than that with $1 \times$ MIC SH treatment $(p<0.05, \mathrm{n}=4)$ as compared with the control group. In the 7th day (Figure 1C), both biofilms treated by $1 \times$ MIC and $2 \times$ MIC SH have exceedingly significant differences $(p=0.000, \mathrm{n}=4)$.

Figure 2 shows controls of LFX and SH + LFX on biofilm formation of Pseudomonas aeruginosa. In Figure 2A (day 1), LFX alone has obviously curbed biofilm formation, and except for the sample treated by $1 / 2 \times$ MIC LFX $(p<0.05, n=4)$, the differences in other two cases by $1 \times$ MIC and $2 \times$ MIC LFX are extremely significant ( $p=0.000, \mathrm{n}=4$ ) as compared with control. After combination with $\mathrm{SH}$, the biofilm growths are all lower than the corresponding ones treated by LFX alone $(p=0.000, \mathrm{n}=4)$. 
Similar results also appear in Figure 2B (day 3). With the extension of biofilm development, the synergistic effects in Figure 2C (day 7) are even more notable.

Figure 1. The effect of $\mathrm{SH}$ alone on the biofilm growth of Pseudomonas aeruginosa. $*, p<0.05 ; * *, p<0.01 ; * * *, p=0.000 ; \mathrm{n}=4$. SH: sodium houttuyfonate.
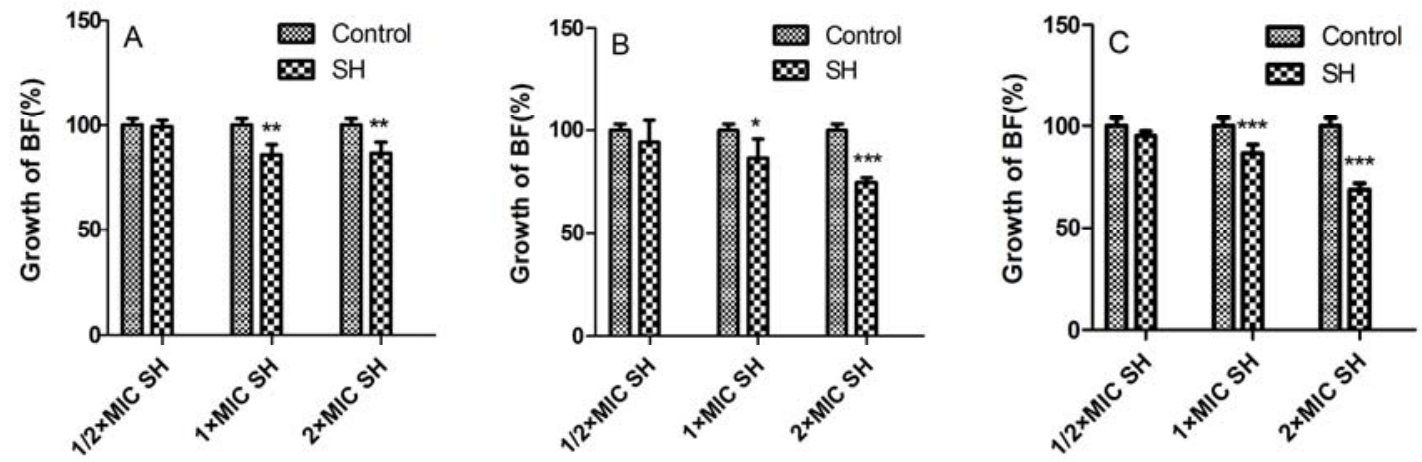

Figure 2. The synergistic effect of SH and LFX on the biofilm formation of Pseudomonas aeruginosa. ${ }^{*}, p<0.05 ; * *, p<0.01 ; * * *, p=0.000 ; \mathrm{n}=4$. SH: sodium houttuyfonate; LFX: levofloxacin.
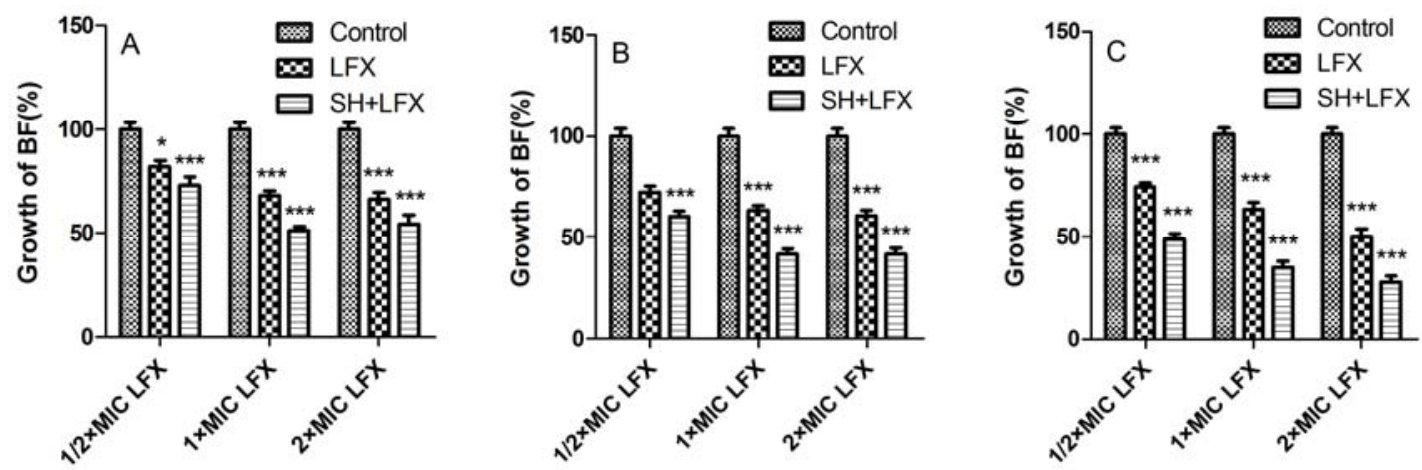

From the above results, it could be observed that the effect of SH alone on biofilm control is limited, as even the maximum dosage of $\mathrm{SH}(=2 \times \mathrm{MIC})$ can merely restrain about $30 \%$ biofilm growth (Figure 1C). This may be related to the fact that $\mathrm{SH}$ is more active on Gram-positive $\left(\mathrm{G}^{+}\right)$bacteria than Gram-negative $\left(\mathrm{G}^{-}\right)$ones [19]. However, the combination of SH with LFX exhibits strong (more than $50 \%$ biofilm growth in Figure $2 \mathrm{C}$ ) and persistent (7 days) inhibition of biofilm. More importantly, $\mathrm{SH}$ increases the biofilm suppression in combination with LFX. That is to say, phytoanticipins may have a nice synergistic effect with microbial antibiotics. Next, it was of interest to probe into the synergistic effect of SH and LFX on alginate production.

\subsection{Alginate Removal by SH Alone and in Combination with LFX}

Alginate is an acetylated polymer, composed of non-repetitive monomers of $\beta$-1,4-linked L-guluronic and D-mannuronic acids [22]. Until now, it has been assumed to be one of the most critical factors contributing to antibiotic tolerance and immune evasion, and not just playing a structural role [23-25]. Recently, Mann and Wozniak published a comprehensive review on this aspect [26]. Thus, it is necessary to investigate the effect of SH on alginate inhibition in combination of LFX. 
Figure 3 shows the effect of only $\mathrm{SH}$ on alginate production of Pseudomonas aeruginosa biofilm. It is interesting to observe that: (i) alginate is still produced on the first day after treatment of $1 / 2 \times$ MIC $\mathrm{SH}$ and $1 \times \mathrm{MIC} \mathrm{SH}$, and its concentration decreased dramatically to about $1 / 3(p<0.01, \mathrm{n}=4)$ after increasing the dosage of SH to $2 \times \mathrm{MIC}$ (Figure 3A); (ii) after three-day medication, the alginate concentration is still a little higher than the control concentration when using $1 / 2 \times \mathrm{MIC} \mathrm{SH}$, but then becomes lower and has statistically significant differences $(p<0.05$ and $p=0.000, \mathrm{n}=4)$ with the respective dosages of $1 \times \mathrm{MIC} \mathrm{SH}$ and $2 \times \mathrm{MIC} \mathrm{SH} \mathrm{(Figure} \mathrm{3B);} \mathrm{and} \mathrm{(iii)} \mathrm{after} \mathrm{administration} \mathrm{until} \mathrm{the}$ seventh day, the inhibitions of alginate become more and more remarkable with the addition of $\mathrm{SH}$ from $1 / 2 \times$ MIC to $2 \times$ MIC, as the alginate productions are reducing continuously $(p<0.05, p=0.000$ and $p=0.000, \mathrm{n}=4$, Figure $3 \mathrm{C}$ ).

Figure 3. The effect of $\mathrm{SH}$ alone on alginate production of Pseudomonas aeruginosa biofilm. *, $p<0.05 ; * *, p<0.01 ; * * *, p=0.000 ; \mathrm{n}=4$. SH: sodium houttuyfonate.
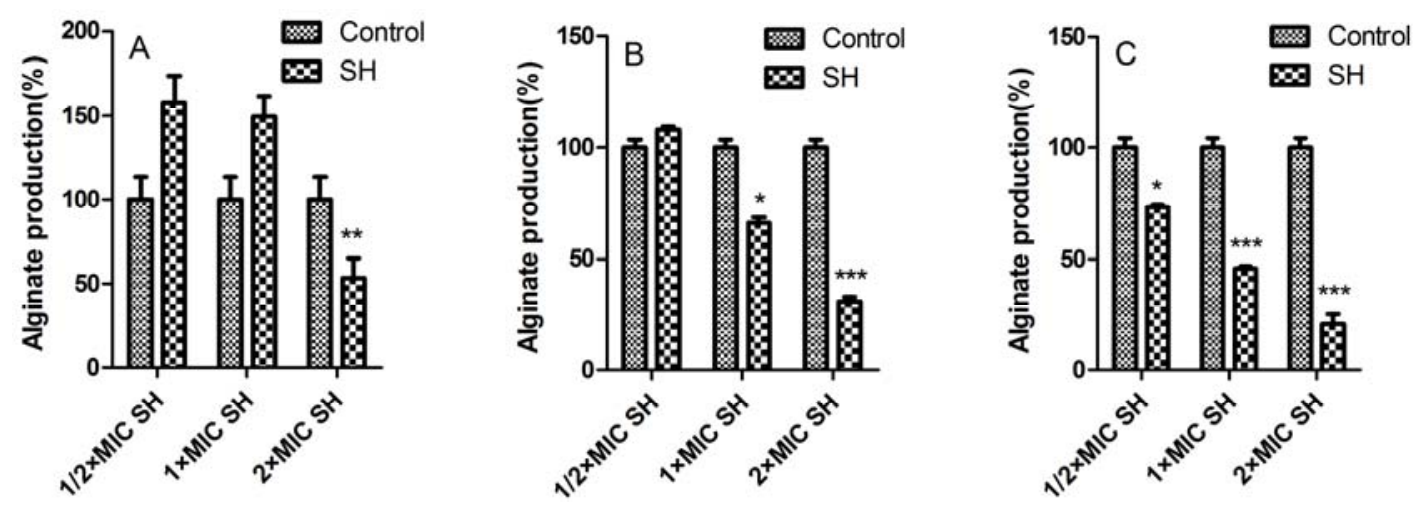

In Figure 4, the synergistic effect of SH and LFX is evaluated. When treated by LFX alone, the situations are similar to those in Figure 3. The suppressions of alginate production basically have no statistical significance (Figure 4A,B) as compared with those in control group, which is changed until biofilm is treated for seven days ( $p<0.01, \mathrm{n}=4$, Figure 4C). However, the synergistic effects become visible with $1 \times$ MIC and $2 \times$ MIC SH in day $1(p<0.05, \mathrm{n}=4$, Figure $4 \mathrm{~A})$, and the effects become even more prominent in day 3 in all three dosage $(p=0.000, \mathrm{n}=4$, Figure $4 \mathrm{~B})$. The trend is kept to the day 7 ( $p=0.000, \mathrm{n}=4$, Figure $4 \mathrm{C})$.

Similar to the case in Figure 1, SH alone has a mild influence on alginate production (Figure 3). But with the addition of LFX, their synergistic effects gradually impose great limitations on alginate production (Figure 4). As known, alginate is secreted by bacteria to form an extracellular matrix, and finally encase the bacteria to help them hide from the toxic effects of antimicrobial agents. In Figures $2 \mathrm{C}$ and $4 \mathrm{C}$, the reduction of biofilm growth leads to the stagnancy or loss of alginate production, inferring that alginate may at least partly participate in biofilm formation. 
Figure 4. The synergistic effect of $\mathrm{SH}$ and LFX on the alginate production of Pseudomonas aeruginosa biofilm. *, $p<0.05 ; * *, p<0.01 ; * * *, p=0.000 ; \mathrm{n}=4$. SH: sodium houttuyfonate; LFX: levofloxacin.
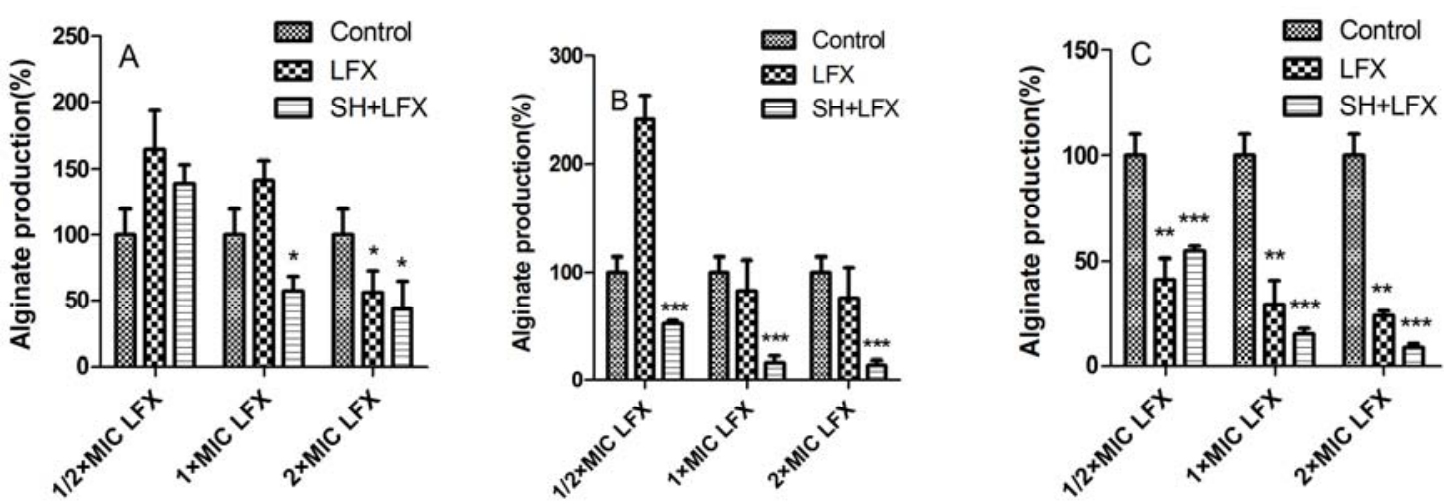

\subsection{In Vitro Antimicrobial Role of SH and Its Synergistic Activity with LFX}

It can be observed that the growth of biofilm decreases from $99 \pm 2.77 \%$ after one-day administration of $1 / 2 \times$ MIC SH to $69 \pm 3.07 \%$ after seven-day administration of $2 \times \mathrm{MIC}$ SH in Figure 1, and the alginate production lowers from $158 \pm 15.9 \%$ after one-day medication to $21 \pm 4.61 \%$ after seven-day medication in Figure 3. Thus, SH exhibits a certain antimicrobial activity in vitro.

It can be seen that the biofilm growth loses about $45 \%$ between one-day therapy of $1 / 2 \times$ MIC $\mathrm{SH}+1 / 2 \times$ MIC LFX and seven-day therapy of $1 / 2 \times$ MIC SH $+2 \times$ MIC LFX in Figure 2, and the alginate production drops about $131 \%$ between one-day treatment of $1 / 2 \times \mathrm{MIC} \mathrm{SH}+1 / 2 \times \mathrm{MIC}$ LFX and seven-day treatment of $1 / 2 \times$ MIC SH $+2 \times$ MIC LFX in Figure 4. Therefore, SH has a very intense synergy with LFX in the restriction of biofilm growth and alginate production.

From Figure 1 to Figure 4, it can be manifested that: (i) both indexes, i.e., growth of biofilm and alginate production, are dosage-dependent, because they display a decreasing tendency with the addition of LFX or SH; (ii) both items are time-dependent, as the suppression effects become better with the elongation of administration from day 1 to day 7. As for the synergistic effect of SH and LFX, it is also interesting to note that the alginate production effects are not synchronous with those of biofilm growth, which can be inferred from two aspects: (i) the alginate production still increases in comparison with control when employing a lower dosage of $1 / 2 \times \mathrm{MIC} \mathrm{LFX}+1 / 2 \times \mathrm{MIC} \mathrm{SH}$, which is more noteworthy in the first day of dosage $(=139 \pm 13.52 \%$, Figure $4 \mathrm{~A})$, suggesting no effective suppression of the combined antibiotics on alginate production; in the same period, the growth of biofilm is $73 \pm 3.9 \%$ by SH in combination of LFX, showing a certain inhibition (Figure $2 \mathrm{~A}$ ); (ii) the alginate production is only $8 \pm 1.71 \%$ when using a higher dosage of $2 \times \mathrm{MIC}$ LFX $+1 / 2 \times \mathrm{MIC}$ SH after seven days treatment (Figure 4C), while the growth of biofilm accounts for $28 \pm 3.06 \%$ with the same time-span (Figure 2C), higher than that of alginate production. These discrepancies may be caused by following reasons: (i) in the stage of initial attachment (the first day), some planktonic bacteria are suppressed or killed by antibiotic alone or in combination, leading to biofilm reduction, while other irreversibly-attached bacteria begin to synthesize and secrete alginate in large amounts; (ii) the alginate is mainly produced by the above bacteria which have enough nutrients to continue their 
metabolism, but the inner bacteria tend to remain in a dormant mode due to limited nutrition, causing them to escape the destruction by combined antibiotics [5]. As a result, when the alginate production is restrained, the encased bacteria mentioned above are exposed to be killed, while the continuous killing of the inner bacteria still needs time and re-addition of antibiotics in combination; and (iii) we cannot rule out other factors, e.g., quorum sensing (QS), that might influence biofilm formation except the production of alginate, which is still under investigation in our lab.

\subsection{Observation of Pseudomonas aeruginosa by Fluorescence Microscope}

Figure 5 presents the fluorescence microscope images of the bacteria. The FAD-PI dyed bacteria are treated by SH alone, LFX alone, and SH + LFX. Living bacteria exhibit green and dead bacteria show red.

Figure 5. Observation of bacterial viability by BX51 fluorescence microscope $(\times 400)$ under the treatment of no drugs, $2 \times \mathrm{MIC} \mathrm{SH}, 2 \times \mathrm{MIC} \mathrm{LFX}$, and $1 / 2 \times \mathrm{MIC} \mathrm{SH}+2 \times \mathrm{MIC}$ LFX, and the exposure of $100 \mu \mathrm{g} / \mathrm{mL}$ FAD for $5 \mathrm{~min}$ and $60 \mu \mathrm{g} / \mathrm{mL}$ PI for $5 \mathrm{~min}$. Green: living cells stained by FAD; Red: dead cells stained by PI. Other conditions are given in Section 3.7.

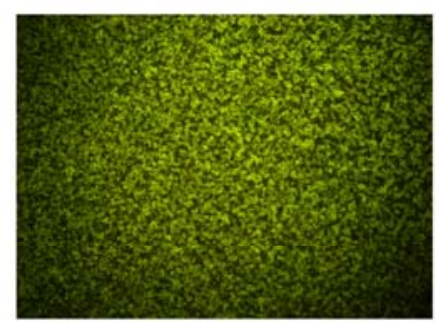

Control

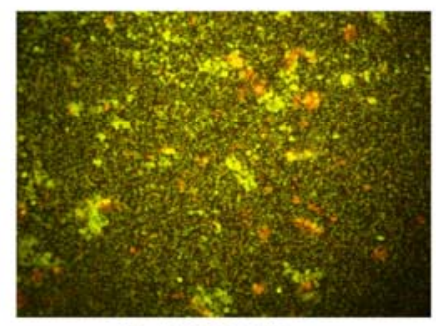

$2 \times$ MIC LFX

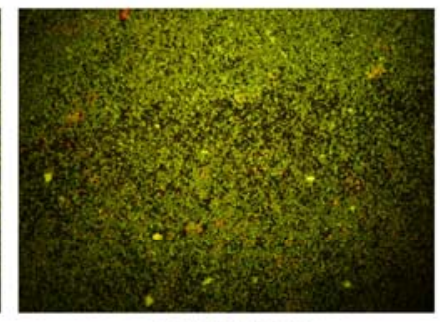

$2 \times$ MIC SH

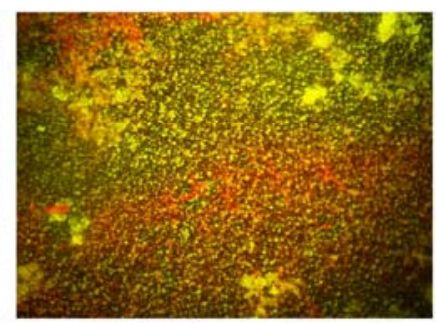

$1 / 2 \times$ MIC SH+2 $\times$ MIC LFX

In Figure 5, it can be observed that: (i) living bacteria are scattered in control group as all cells are seen to be green; (ii) most bacteria are alive and less are dead as most cells are recorded to be green with less red in $2 \times$ MIC SH group and $2 \times$ MIC LFX group, however, the bacteria viability in the $2 \times$ MIC LFX group is notably less than that in $2 \times$ MIC SH group which has more red cells in view; and (iii) less bacteria in the $1 / 2 \times$ MIC SH $+2 \times$ MIC LFX group are alive than those in the SH alone group and LFX alone group as more red dots appear in the image. From the four images, the quantities of dead cell exhibit an increasing tendency, indicating the antimicrobial effects promoted by $\mathrm{SH}$ and LFX in combination. 


\subsection{Observation of Pseudomonas aeruginosa Biofilm Morphology by SEM}

Figure 6 shows is the morphology of the bacterial biofilm by SEM. In the control group, the bacteria are largely covered by mucous substances which are mainly composed of alginate, and dense cells are seen to exist in the whole view, which is similar to that in the control group in Figure 5. In the $2 \times$ MIC SH group, the mucous substances are largely removed, but the bacteria are not completely inhibited, indicating that the $\mathrm{SH}$ is mainly suppressing the production of mucuous substances. In terms of the results that the alginate production (\%) is about $21 \%$ (Figure $3 \mathrm{C}$ ) after the treatment of $2 \times$ MIC $\mathrm{SH}$, it is obvious that the alginate is the main substance in the mucuous substances and $\mathrm{SH}$ largely targets the alginate. In the $2 \times$ MIC LFX group, both mucous substance and bacteria are dramatically decreased, however, there are still some matrix substances left, revealing the relative strong antipathogenic of LFX on alginate and encased bacteria. In the $1 / 2 \times \mathrm{MIC} \mathrm{SH}+2 \times \mathrm{MIC}$ LFX group, both mucous substance and bacteria are wiped out further compared with those in the $2 \times$ MIC LFX group, reflecting that $\mathrm{SH}$ can enhance the suppressive effects of LFX on alginate to kill the bacteria under the mucus substances, i.e., SH and LFX have better synergistic effects on biofilm removal.

Figure 6. Observation of bacterial biofilm morphology by SEM.

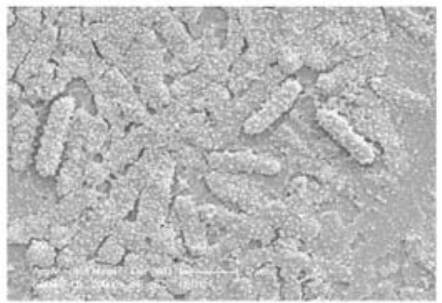

Control

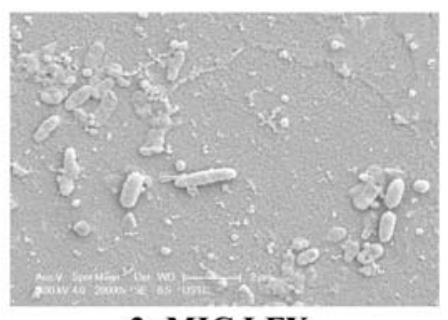

$2 \times$ MIC LFX

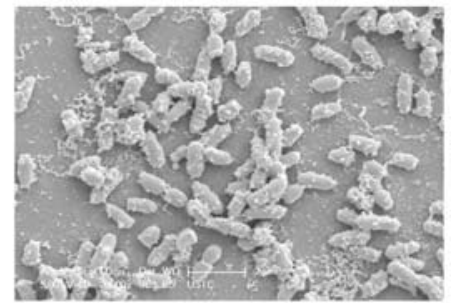

$2 \times$ MIC SH

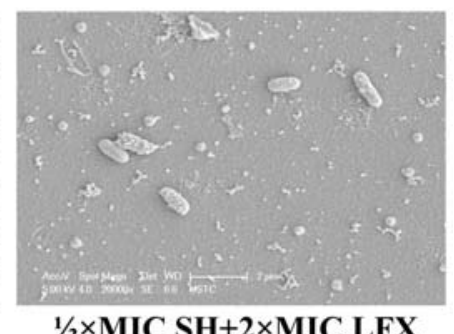

\section{Experimental}

\subsection{Strains and Materials}

Pseudomonas aeruginosa ATCC27853, sodium houttuyfonate (SH, Figure 7) and levofloxacin (LFX) were all obtained from the National Institute for the Control of Pharmaceutical and Biological Products (NICPBP, Beijing, China). The LB, MH and TSB media were purchased from Aoboxing Bio-tech Co., Ltd. (Beijing, China). Crystal violet solution was purchased from bioMérieux (Lyon, France). The alginate standards, fluorescein di-o-acetate (FDA) and propidium iodide (PI) were obtained from Sigma (St. Louis, MO, USA). 
Figure 7. The chemical structures of sodium houttuyfonate (SH) and houttuynin (decanoyl acetaldehyde).

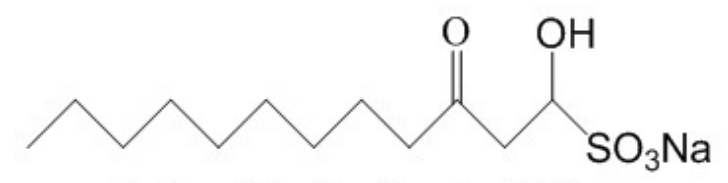

Sodium Houttuyfonate (SH)

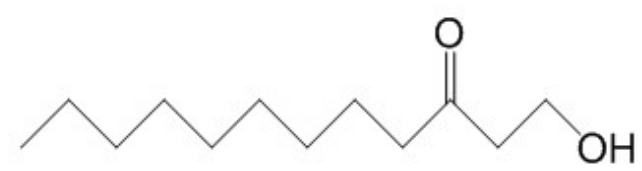

Houttuynin (decanoyl acetaldehyde)

\subsection{Cultivation of Pseudomonas aeruginosa}

Pseudomonas aeruginosa ATCC27853 was inoculated in LB broth, cultivated in a constant-temperature shaker GLY (Fuma, Shanghai, China) of $220 \mathrm{rpm}$ for $6 \mathrm{~h}$ at constant $37{ }^{\circ} \mathrm{C}$. Then the bacteria were harvested by GL-20G- II high-speed refrigerated centrifuge (Fuma) at 2,000 r/min for $10 \mathrm{~min}$. The supernate was discarded, and the precipitate was resuspended with $\mathrm{pH} 7.2$ phosphate-buffered saline (PBS), centrifuged again at 2,000 r/min for $10 \mathrm{~min}$. The collection was mixed with PBS $\mathrm{pH} 7.2$, and adjusted to $2 \times 10^{5} \mathrm{CFU} / \mathrm{mL}$ by the microdilution method.

\subsection{Preparation of SH and LFX Solutions}

Both stock solutions of SH and LFX were prepared freshly in MH broth and filtered through a $0.22 \mu \mathrm{m}$ Millipore filter (Millipore, Billerica, MA, USA), and their concentrations were adjusted to 160 and $32 \mu \mathrm{g} / \mathrm{mL}$, respectively. In the MIC determination experiments, SH was twofold-diluted into ten concentration gradients by $\mathrm{MH}$ medium at a final concentration from 0.03125 to $16 \mathrm{mg} / \mathrm{mL}$, and LFX was diluted to a final concentration ranging from 0.0625 to $32 \mu \mathrm{g} / \mathrm{mL}$.

\subsection{MIC Measurement}

Both SH and LFX were added into 96-well plates with eight wells in duplicate for each concentration gradient, and each well had both $100 \mu \mathrm{L}$ either SH or LFX solution and $100 \mu \mathrm{L}$ inoculum with the final concentration of $1 \times 10^{5} \mathrm{CFU} / \mathrm{mL}$. The plates were incubated for $24 \mathrm{~h}$ at $37^{\circ} \mathrm{C}$, and then the bacteria were inoculated on nutrient agar medium and incubated for another $24 \mathrm{~h}$ at $37^{\circ} \mathrm{C}$. The MIC was determined as the minimum drug dilution with no growth of bacteria on plates. Each MIC assay was performed in triplicate. The checkerboard microdilution method was adopted for antimicrobial assay of SH and LFX in synergy. The concentrations of combined antibiotics were adjusted from $1 / 8 \times$ MIC to $2 \times \mathrm{MIC}$, and $100 \mu \mathrm{L}$ drugs were added into each well. A volume of $100 \mu \mathrm{L} /$ well inoculum was mixed with the drugs to the final concentration of $1 \times 10^{5} \mathrm{CFU} / \mathrm{mL}$. Then, the setup was incubated at $37^{\circ} \mathrm{C}$ for $24 \mathrm{~h}$ to observe the growth of bacteria, which was detected by a 318-microplate reader (Sanco Instruments, Shanghai, China). The control had medium and inoculum without any antimicrobial agents. Each test was performed in triplicate [27]. 


\subsection{Effects of SH Alone and SH + LFX in Combination on Anti-Biofilm Formation}

Ten groups were tested: control, $1 / 2 \times \mathrm{MIC} \mathrm{SH}, 1 \times \mathrm{MIC} \mathrm{SH}, 2 \times \mathrm{MIC} \mathrm{SH}, 1 / 2 \times$ MIC LFX, $1 \times$ MIC LFX, $2 \times$ MIC LFX, $1 / 2 \times$ MIC SH $+1 / 2 \times$ MIC LFX, $1 / 2 \times$ MIC SH $+1 \times$ MIC SH, $1 / 2 \times$ MIC $\mathrm{SH}+2 \times$ MIC LFX (Figures 1 and 2). In a 96-well plate, each was added with $200 \mu \mathrm{L}$ Pseudomonas aeruginosa suspension, incubated at $37{ }^{\circ} \mathrm{C}$ with no shaking. After $24 \mathrm{~h}$, the strain suspension was discarded, and the planktonic microbes were washed by PBS, inoculated into TSB medium with the above concentrations of antibiotics. The control was cultivated in the medium with no antibiotics. According to the description of O'Toole [28], the medium were exchanged for a new one with antibiotics every other day. At the end of the 1 st, $3 \mathrm{rd}$ and 7 th day, $4{ }^{\circ} \mathrm{C}$ PBS was employed to wash the planktonic bacteria on the 96-well plate. Then, each well was added into $200 \mu \mathrm{L} 1 \%$ crystal violet solution for $20 \mathrm{~min}$. After they were rinsed with de-ionized water till no crystal violet was visible, the wells were dried and 95\% alcohol added to destain. The destained samples at the end of 1st, 3rd and 7 th day of medications were transferred to a cuvette and diluted with $95 \%$ alcohol $(3 \mathrm{~mL})$ to determine the $\mathrm{OD}$ value $\left(\mathrm{OD}_{\mathrm{b} \text {-sample }}\right)$ at the wavelength of $570 \mathrm{~nm}$. The corresponding $\mathrm{OD}$ values of negative control $\left(\mathrm{OD}_{\mathrm{b} \text {-control }}\right)$, consisting of biofilm without any medications, were treated as the above steps and set as $100 \%$ at the end of 1 st, 3rd and 7 th day of medications. Then, the growth of biofilm (\%) could be calculated for each group as follows:

$$
\text { Growth of biofilm }(\%)=\frac{\mathrm{OD}_{\text {b-sample }}}{\mathrm{OD}_{\text {b-control }}} \times 100 \%
$$

and each calculation was processed in triplicate.

\subsection{Effects of SH Alone and SH + LFX in Combination on Anti-Alginate Production}

There were also ten groups, the same as those in Section 3.5. The sterile cover-glass carrier was put into the 6-well culture plate, and then TSB medium $(2 \mathrm{~mL})$ and strain suspension $(0.2 \mathrm{~mL})$ were added. After incubating for 1 day at $37^{\circ} \mathrm{C}$, the medium was discarded, and the cover-glass was taken out to wash the planktonic bacteria out with sterile PBS. The cover-glass was then put into the well again. The antibiotic groups were given medium containing antibiotics, while the negative control used medium without antibiotics. All the above manipulations were repeated in each $24 \mathrm{~h}$. At the end of the 1st, 3rd and 7th day of medication, the cover-glasses were fetched out to rinse the planktonic bacteria by PBS. Then, the cover-glasses were put into test tube with additions of PBS $(6 \mathrm{~mL})$, sulphuric acid and sodium borate $\left(1.2 \mathrm{~mL}\right.$ each), boiled for $5 \mathrm{~min}$, and placed at $4{ }^{\circ} \mathrm{C} .1 \%$ Hydroxybiphenyl $(20 \mu \mathrm{L})$ was added to the glass for colorization, and vibrated for $30 \mathrm{~min}$ by ultrasound. The OD values at the end of $1 \mathrm{st}, 3 \mathrm{rd}$ and 7 th day of medications were recorded at the wavelength of $570 \mathrm{~nm}\left(\mathrm{OD}_{\text {a-sample }}\right)$. According to the above treatments, the corresponding $\mathrm{OD}$ values of negative control $\left(\mathrm{OD}_{\mathrm{a}-\mathrm{control}}\right)$, consisting of alginate without any medications, were set as $100 \%$, and then the alginate production (\%) could be calculated for each group as follows:

$$
\text { Alginate production }(\%)=\frac{\mathrm{OD}_{\text {a-sample }}}{\mathrm{OD}_{\text {a-control }}} \times 100 \%
$$

and each calculation was conducted in triplicate. 


\subsection{Fluorescence Microscopy of Bacteria}

The carrier disposition was similar to that described in Section 3.6. After the glass was removed and the planktonic bacteria rinsed with PBS at the end of the 7th day of medication, it was stained with fluorescein diacetate-propidium iodide (FDA-PI) fluorescent dyes to observe the vitality of dispersed bacteria, in which FDA concentration was $100 \mu \mathrm{g} / \mathrm{mL}$, and PI concentration was $60 \mu \mathrm{g} / \mathrm{mL}$. The bacteria were first stained by FDA for $5 \mathrm{~min}$, and then with PI for $5 \mathrm{~min}$. The dead bacteria were stained as red by PI, while the alive ones were as green by FDA. A BX51 fluorescence microscope was used (Olympus, Tokyo, Japan) to record the results.

\subsection{Scanning Electric Microscope of Biofilm Morphology}

The biofilm morphology was observed by silver-staining on a SEM (Sirion200, FEI, Columbia, MD, USA).

\section{Conclusions}

A phytoanticipin derivative, sodium houttuyfonate, was adopted to investigate its synergistic effects with levofloxacin on the suppression of Pseudomonas aeruginosa biofilm. We separately evaluated the inhibitions by SH alone and LFX alone on biofilm formation and alginate production, and the synergistic effect of SH with LFX on biofilm formation and alginate production. The results demonstrate that $\mathrm{SH}$ has robust synergistic activity with LFX, and can dramatically decrease the amount of bacteria by effective inhibition of biofilm formation and alginate production. Consequently, phytoanticipins represent a promising alternative to coordinate with microbial antibiotics, playing an even greater role in anti-biofilm treatment.

\section{Acknowledgments}

This work was supported by the National Natural Science Foundation of China (No. 81173629).

\section{Conflict of interest}

The authors declare no conflict of interest.

\section{References}

1. Costerton, J. Introduction to biofilm. Int. J. Antimicrob. Agents 1999, 11, 217-221.

2. Hall-Stoodley, L.; Costerton, J.W.; Stoodley, P. Bacterial biofilms: From the natural environment to infectious diseases. Nat. Rev. Microbiol. 2004, 2, 95-108.

3. Kirketerp-Møller, K.; Jensen, P.Ø.; Fazli, M.; Madsen, K.G.; Pedersen, J.; Moser, C.; Tolker-Nielsen, T.; Høiby, N.; Givskov, M.; Bjarnsholt, T. Distribution, organization, and ecology of bacteria in chronic wounds. J. Clin. Microbiol. 2008, 46, 2717-2722.

4. Bjarnsholt, T.; Jensen, P.O.; Fiandaca, M.J.; Pedersen, J.; Hansen, C.R.; Andersen, C.B.; Pressler, T.; Givskov, M.; Hoiby, N. Pseudomonas aeruginosa biofilms in the respiratory tract of cystic fibrosis patients. Pediatr. Pulmonol. 2009, 44, 547-558. 
5. Harmsen, M.; Yang, L.; Pamp, S.J.; Tolker-Nielsen, T. An update on pseudomonas aeruginosa biofilm formation, tolerance, and dispersal. FEMS Immunol. Med. Microbiol. 2010, 59, 253-268.

6. Carneiro, V.A.; Santos, H.S.; Arruda, F.V.; Bandeira, P.N.; Albuquerque, M.R.; Pereira, M.O.; Henriques, M.; Cavada, B.S.; Teixeira, E.H. Casbane diterpene as a promising natural antimicrobial agent against biofilm-associated infections. Molecules 2011, 16, 190-201.

7. Hengzhuang, W.; Wu, H.; Ciofu, O.; Song, Z.; Hoiby, N. In vivo pharmacokinetics/ pharmacodynamics of colistin and imipenem in pseudomonas aeruginosa biofilm infection. Antimicrob. Agents Chemother. 2012, 56, 2683-2690.

8. Saleh, S.; Taha, M.O.; Haddadin, R.N.; Marzooqa, D.; Hodali, H. Preparation of silver- and zinc-doped mullite-based ceramics showing anti-bacterial biofilm properties. Molecules 2011, 16, 2862-2870.

9. Saleh, S.; Sweileh, B.; Taha, S.O.; Mahmoud, R.; Taha, M.O. Preparation of polyester-based metal-cross linked polymeric composites as novel materials resistant to bacterial adhesion and biofilm formation. Molecules 2011, 16, 933-950.

10. Hengzhuang, W.; Wu, H.; Ciofu, O.; Song, Z.; Hoiby, N. Pharmacokinetics/pharmacodynamics of colistin and imipenem on mucoid and nonmucoid pseudomonas aeruginosa biofilms. Antimicrob. Agents Chemother. 2011, 55, 4469-4474.

11. VanEtten, H.D.; Mansfield, J.W.; Bailey, J.A.; Farmer, E.E. Two classes of plant antibiotics: Phytoalexins versus "phytoanticipins". Plant Cell 1994, 6, 1191-1192.

12. Dixon, R.A. Natural products and plant disease resistance. Nature 2001, 411, 843-847.

13. Zeng, X.; Liu, X.; Bian, J.; Pei, G.; Dai, H.; Polyak, S.W.; Song, F.; Ma, L.; Wang, Y.; Zhang, L. Synergistic effect of 14-alpha-lipoyl andrographolide and various antibiotics on the formation of biofilms and production of exopolysaccharide and pyocyanin by pseudomonas aeruginosa. Antimicrob. Agents Chemother. 2011, 55, 3015-3017.

14. Kosuge, T. Structure of an antimicrobial substance isolated from houttuynia cordata thunb. J. Pharm. Sac. Jpn. 1952, 72, 12271-12275.

15. Liu, G.; Xiang, H.; Tang, X.; Zhang, K.; Wu, X.; Wang, X.; Guo, N.; Feng, H.; Wang, G.; Liu, L.; et al. Transcriptional and functional analysis shows sodium houttuyfonate-mediated inhibition of autolysis in staphylococcus aureus. Molecules 2011, 16, 8848-8865.

16. Zhou, Y.; Zhou, M.; Zhang, D.; Zhang, H.; Zhang, L. Immune response of aa broilers to ibv h120 vaccine and sodium new houttuyfonate. Res. Vet. Sci. 2010, 89, 373-377.

17. Pan, P.; Wang, Y.J.; Han, L.; Liu, X.; Zhao, M.; Yuan, Y.F. Effects of sodium houttuyfonate on expression of nf-kappab and mcp-1 in membranous glomerulonephritis. J. Ethnopharmacol. 2010, 131, 203-209.

18. Gao, J.P.; Chen, C.X.; Wang, Y.; Lu, J.; Gu, W.L. Effect of sodium houttuyfonate on myocardial hypertrophy in mice and rats. J. Pharm. Pharmacol. 2009, 61, 677-683.

19. Ye, X.; Li, X.; Yuan, L.; Ge, L.; Zhang, B.; Zhou, S. Interaction of houttuyfonate homologues with the cell membrane of gram-positive and gram-negative bacteria. Colloids Surf. A Physicochem. Eng. Aspects 2007, 301, 412-418.

20. Johnson, D.; Jones, R. In-vitro activity of a combination of two oral beta-lactams (cefpodoxime and amoxycillin) against streptococcus pneumoniae isolates with reduced susceptibilities to penicillin. J. Antimicrob. Chemother. 1998, 42, 555-557. 
21. Sauer, K.; Camper, A.K.; Ehrlich, G.D.; Costerton, J.W.; Davies, D.G. Pseudomonas aeruginosa displays multiple phenotypes during development as a biofilm. J. Bacteriol. 2002, 184, 1140-1154.

22. Evans, L.R.; Linker, A. Production and characterization of the slime polysaccharide of pseudomonas aeruginosa. J. Bacteriol. 1973, 116, 915-924.

23. Simpson, J.A.; Smith, S.E.; Dean, R.T. Alginate inhibition of the uptake of pseudomonas aeruginosa by macrophages. J. Gen. Microbiol. 1988, 134, 29-36.

24. Simpson, J.A.; Smith, S.E.; Dean, R.T. Scavenging by alginate of free radicals released by macrophages. Free Radic. Biol. Med. 1989, 6, 347-353.

25. Simpson, J.; Smith, S.; Dean, R. Alginate may accumulate in cystic fibrosis lung because the enzymatic and free radical capacities of phagocytic cells are inadequate for its degradation. Biochem. Mol. Biol. Int. 1993, 30, 1021-1034.

26. Mann, E.E.; Wozniak, D.J. Pseudomonas biofilm matrix composition and niche biology. FEMS Microbiol. Rev. 2012, 36, 893-916.

27. Zhou, Y.; Wang, G.; Li, Y.; Liu, Y.; Song, Y.; Zheng, W.; Zhang, N.; Hu, X.; Yan, S.; Jia, J. In vitro interactions between aspirin and amphotericin b against planktonic cells and biofilm cells of candida albicans and c. Parapsilosis. Antimicrob. Agents Chemother. 2012, 56, 3250-3260.

28. O’Toole, G.A. Microtiter dish biofilm formation assay. J. Vis. Exp. 2011, 47, e2437.

Sample Availability: Samples of Sodium Houttuyfonate are available from the authors.

(C) 2012 by the authors; licensee MDPI, Basel, Switzerland. This article is an open access article distributed under the terms and conditions of the Creative Commons Attribution license (http://creativecommons.org/licenses/by/3.0/). 